\section{Tracking and Regulation Control of an Underactuated Surface Vessel With Nonintegrable Dynamics}

\author{
A. Behal, D. M. Dawson, W. E. Dixon, and Y. Fang
}

\begin{abstract}
In this note, a continuous, time-varying tracking controller is designed that globally exponentially forces the position/orientation tracking error of an underactuated surface vessel to a neighborhood about zero that can be made arbitrarily small [i.e., global uniformly ultimately boundedness (GUUB)]. The result is facilitated by fusing a filtered tracking error transformation with the dynamic oscillator design presented in [6]. We also illustrate that the proposed tracking controller yields a GUUB result for the regulation problem.
\end{abstract}

Index Terms-Lyapunov, nonlinear, surface vessels, tracking, underactuated.

\section{INTRODUCTION}

Over the past decade, many researchers have studied the control problem for underactuated systems with nonintegrable constraints. The majority of this research has targeted nonholonomic systems (i.e., systems with nonintegrable velocity constraints), such as wheeled mobile robots and the general chained-form system (for a survey of research that has targeted tracking and regulation control of nonholonomic systems see [4], [7], [6], [10]-[12], [18], [19], and the references within). For an overview of smooth and nonsmooth tracking/stabilizing controllers for systems that satisfy the nonholonomic constraint, we refer the reader to [2] and [20]. However, motivated by the challenging theoretical aspects and numerous practical applications, researchers have also attacked underactuated systems with nonintegrable dynamics (e.g., surface vessels, twin rotor helicopters, underwater vehicles, V/CTOL aircraft, etc.). For example, in [17], Reyhanoglu et al. provides a detailed discussion on the controllability and the stabilizability of underactuated mechanical systems with nonintegrable dynamics. The conclusion from this discussion is a result similar to Brockett's condition [3] for nonholonomic systems. That is, Reyhanoglu et al. illustrated that underactuated systems with nonintegrable dynamics cannot be asymptotically stabilized by a continuous, time-invariant feedback law. In [13], Pettersen et al. showed that underactuated surface vessels cannot be asymptotically stabilized by continuous time-invariant feedback laws. In addition, Pettersen et al. [13] proposed a time-varying feedback controller for an underactuated surface vessel that contained explicit time-periodic sinusoidal terms (similar in structure to [18]) to obtain local exponential regulation. In [14], Pettersen et al. modified the continuous time-varying feedback law of [13] to design a controller

Manuscript received September 1, 2000; revised January 29, 2001 and November 10, 2001. Recommended by Associate Editor W. Lin. This work was supported in part by the Eugene P. Wigner Fellowship Program of the Oak Ridge National Laboratory (ORNL), managed by UT-Battelle, LLC, for the U.S. Department of Energy (DOE) under Contract DE-AC05-00OR22725, and in part by the U.S. DOE Environmental Management Sciences Program (EMSP) Project ID 82797 at ORNL, ONR Project N00014-00-F-0485 at ORNL, and by the National Science Foundation under Grant DMI-9457967, ONR Grant N00014-99-1-0589, a DOC Grant, and an ARO Automotive Center Grant.

A. Behal, D. M. Dawson, and Y. Fang are with Department of Electrical and Computer Engineering, Clemson University, Clemson, SC 29634-0915 USA (e-mail: abehal@ces.clemson.edu; ddawson@ces.clemson.edu; yfang@ces.clemson.edu).

W. E. Dixon is with Oak Ridge National Laboratory, Oak Ridge, TN 37831 USA (e-mail: dixonwe@ornl.gov).

Publisher Item Identifier S 0018-9286(02)02829-5. that also locally exponentially regulates the position and orientation of an underactuated surface vessel.

In addition to the regulation problem, several controllers have also been proposed for the tracking control problem. Specifically, in [9], Godhavn utilized a continuous time-invariant state feedback controller to achieve global exponential position tracking provided the desired surge velocity is always positive; however, due to the control structure, the orientation of the surface vessel is not controlled. In [15], Pettersen et al. proposed a tracking controller that achieved global exponential practical stability (i.e., global exponential stability of an arbitrarily small neighborhood of the desired trajectory) of an underactuated surface vessel. In [16], Pettersen et al., proposed a continuous time-invariant control law that obtained semi-global exponential position and orientation tracking, provided the desired angular trajectory remains positive. That is, Pettersen et al. proved semiglobal exponential position and orientation tracking for a class of desired trajectories.

In this note, we design a continuous time-varying tracking controller that yields global uniformly ultimately bounded (GUUB) position/orientation tracking. Specifically, we first manipulate a reference model generator and the dynamic model of an underactuated surface vessel into a form that allows a Lyapunov-based control structure to be developed. That is, motivated by the dynamic oscillator designed in [6] by Dixon et al. for wheeled mobile robots, a time-varying dynamic oscillator is constructed that globally exponentially forces the position/orientation tracking error to a neighborhood about zero that can be made arbitrarily small. The new result is facilitated by fusing a filtered tracking error transformation with the dynamic oscillator design. In addition, since the only restriction we place on the desired trajectory is that the reference generator remain bounded, it is straightforward to illustrate that the proposed controller also yields a GUUB result for the regulation problem.

The note is organized as follows. In Section II, we present the kinematic and dynamic model for an underactuated surface vessel and then transform the open-loop tracking dynamics into a more convenient form for the subsequent controller development and the stability analysis. In Section III, we present the proposed GUUB tracking control design. The corresponding closed-loop error system is given in Section IV while the stability analysis is given in Section V. An extension that illustrates that the proposed tracking controller also solves the regulation problem is given in Section VI. Concluding remarks are presented in Section VII.

\section{Kinematic AND Dynamic Model DeVElopment}

\section{A. Model Formulation}

As described in [8], the kinematic equations of motion of the center of mass (COM) for a surface vessel (SV) can be written as follows:

$$
\dot{q}=S(q) v
$$

where the triplet $\dot{q}(t)=\left(\dot{x}_{c}(t), \dot{y}_{c}(t), \dot{\theta}(t)\right)$ represents the time derivative of $q(t)=\left(x_{c}(t), y_{c}(t), \theta(t)\right), x_{c}(t), y_{c}(t)$ denote the Cartesian position of the COM of the SV, $\theta(t)$ represents the orientation of the SV, $S(q)$ is the $3 \times 3$ transformation matrix defined as follows:

$$
S(q)=\left[\begin{array}{ccc}
\cos \theta & -\sin \theta & 0 \\
\sin \theta & \cos \theta & 0 \\
0 & 0 & 1
\end{array}\right]
$$

and the $3 \times 1$ velocity vector $v(t)$ of $(1)$ is defined as

$$
v=\left[\begin{array}{lll}
v_{1} & v_{2} & v_{3}
\end{array}\right]^{T}
$$




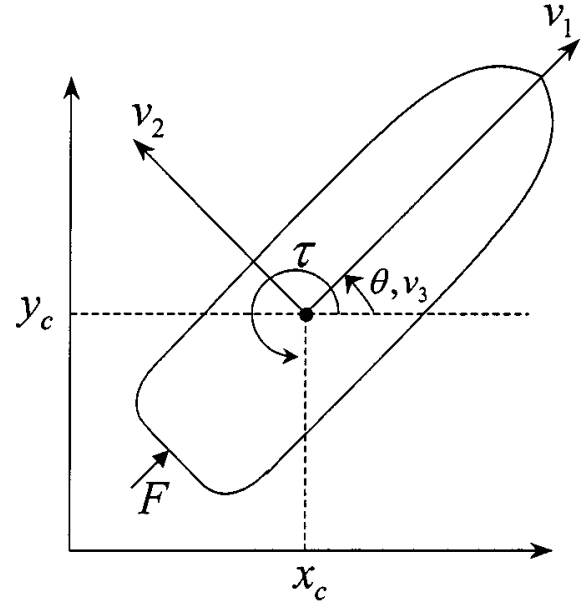

Fig. 1. Actuator diagram for an underactuated surface vessel.

where $v_{1}(t), v_{2}(t)$, and $v_{3}(t)$ denote the surge, sway, and yaw velocities (see Fig. 1) of the SV, respectively. Under the assumptions that i) the body-fixed coordinate axis coincides with the center of gravity (CG), ii) the mass distribution is homogeneous, iii) the hydrodynamic damping terms of order higher than one are negligible, iv) changes in the inertia are negligible, and v) the heave, pitch, and roll modes can be neglected, the dynamic model for a neutrally buoyant SV with two axes of symmetry can be expressed in the following form [8]:

$$
M \dot{v}+D(v) v=\tau_{0}
$$

where $\dot{v}(t)$ denotes the time derivative of $v(t)$ defined in (3), $M$ represents a simplistic $3 \times 3$ constant, diagonal, positive-definite inertia matrix, which is explicitly defined as

$$
M=\left[\begin{array}{ccc}
m & 0 & 0 \\
0 & m & 0 \\
0 & 0 & I_{o}
\end{array}\right]
$$

$m, I_{o}$ represent the mass and inertia of the SV, respectively, $D(v)$ is a 3 $\times 3$ matrix that represents the Centripetal-Coriolis and hydrodynamic damping effects, and is explicitly defined as follows:

$$
D(v)=\left[\begin{array}{ccc}
-X_{v 1} & 0 & -m v_{2} \\
0 & -Y_{v 2} & m v_{1}-Y_{v 3} \\
m v_{2} & -m v_{1}-N_{v 2} & -N_{v 3}
\end{array}\right]
$$

$X_{v 1}, Y_{v 2}, Y_{v 3}, N_{v 2}$, and $N_{v 3}$ denote scalar, constant damping coefficients, and the force-torque control input vector denoted by $\tau_{0}(t)$ is explicitly defined as

$$
\tau_{0}(t)=\left[\begin{array}{lll}
F & 0 & \tau
\end{array}\right]^{T}
$$

where $F(t)$ denotes a control force that is applied to produce a forward thrust, and $\tau(t)$ denotes a torque that is applied about the CG.

In order to simplify the subsequent control development and stability analysis, we first design an outer-loop controller for $F(t)$ and $\tau(t)$ as follows:

$$
F=-X_{v 1} v_{1}+m F_{1}
$$

and

$$
\tau=-N_{v 2} v_{2}-N_{v 3} v_{3}+I_{o} \tau_{1}
$$

${ }^{1}$ In general, the inertias in the surge and sway directions are not equal and the inertia matrix is not diagonal due to hydrodynamic added mass coupling terms. where $F_{1}(t), \tau_{1}(t)$ denote subsequently designed auxiliary control inputs. Based on (3)-(9), we can rewrite the expression for the dynamic model given in (4) as follows:

$$
\left[\begin{array}{c}
\dot{v}_{1} \\
\dot{v}_{2} \\
\dot{v}_{3}
\end{array}\right]=\left[\begin{array}{c}
F_{1}+v_{2} v_{3} \\
\frac{1}{m}\left(Y_{v 2} v_{2}+Y_{v 3} v_{3}\right)-v_{1} v_{3} \\
\tau_{1}
\end{array}\right] .
$$

\section{B. Reference Model Development}

Motivated by the desire to generate a reference model that satisfies the same dynamics as that given in (4), we take the time derivative of $\dot{x}_{c}(t), \dot{y}_{c}(t)$ given in (1) and then use (2), (3), and (10) to obtain the following expression:

$$
\left[\begin{array}{c}
\ddot{x}_{c} \\
\ddot{y}_{c} \\
\dot{\theta}
\end{array}\right]=\left[\begin{array}{c}
F_{1} \cos \theta-\frac{Y_{v 2}}{m}\left(\dot{y}_{c} \cos \theta-\dot{x}_{c} \sin \theta\right) \\
\cdot \sin \theta-\frac{Y_{v 3}}{m} v_{3} \sin \theta \\
F_{1} \sin \theta+\frac{Y_{v 2}}{m}\left(\dot{y}_{c} \cos \theta-\dot{x}_{c} \sin \theta\right) \\
\cdot \cos \theta+\frac{Y_{v 3}}{m} v_{3} \cos \theta \\
v_{3}
\end{array}\right] .
$$

Based on (11), we construct a reference trajectory generator as follows:

$$
\left[\begin{array}{c}
\ddot{x}_{r c} \\
\ddot{y}_{r c} \\
\dot{\theta}_{r}
\end{array}\right]=\left[\begin{array}{c}
F_{1 r} \cos \theta_{r}-\frac{\sin \theta_{r}}{m} \\
\cdot\left(Y_{v 2}\left(\dot{y}_{r c} \cos \theta_{r}-\dot{x}_{r c} \sin \theta_{r}\right)+Y_{v 3} v_{3 r}\right) \\
F_{1 r} \sin \theta_{r}+\frac{\cos \theta_{r}}{m} \\
\cdot\left(Y_{v 2}\left(\dot{y}_{r c} \cos \theta_{r}-\dot{x}_{r c} \sin \theta_{r}\right)+Y_{v 3} v_{3 r}\right) \\
v_{3 r}
\end{array}\right]
$$

where the triplet $\left(x_{r c}(t), y_{r c}(t), \theta_{r}(t)\right)$ represent the Cartesian position and orientation of the reference SV, respectively, and $F_{1 r}(t)$, $v_{3 r}(t)$ denote reference input signals. It is assumed that the reference model is constructed such that $x_{r c}(t), y_{r c}(t), \theta_{r}(t), \dot{x}_{r c}(t), \dot{y}_{r c}(t)$, $\dot{\theta}_{r}(t), \ddot{x}_{r c}(t), \ddot{y}_{r c}(t), \dot{v}_{3 r}(t), F_{1 r}(t) \in \mathcal{L}_{\infty}$ where $\dot{v}_{3 r}(t)$ denotes the time derivative of $v_{3 r}(t)$ defined in (12). Note that the reference orientation is generated by a reference velocity input rather than a reference force or torque input to facilitate the subsequent stability analysis.

\section{Open-Loop Error System Formulation}

To rewrite the open-loop tracking error system in a more convenient form, we define the following global invertible transformation ${ }^{2}$

$$
\begin{gathered}
{\left[\begin{array}{c}
w \\
z_{1} \\
z_{2}
\end{array}\right]=\left[\begin{array}{ccc}
-\tilde{\theta} \cos \theta+2 \sin \theta & -\tilde{\theta} \sin \theta-2 \cos \theta & 2 \frac{Y_{v 3}}{m} \\
0 & 0 & 1 \\
\cos \theta & \sin \theta & 0
\end{array}\right]} \\
\\
\cdot\left[\begin{array}{c}
r_{x} \\
r_{y} \\
\tilde{\theta}
\end{array}\right]
\end{gathered}
$$

where $w(t)$ is an auxiliary scalar error variable, $z(t)=\left[\begin{array}{ll}z_{1}(t) & z_{2}(t)\end{array}\right]^{T}$ is a $2 \times 1$ auxiliary tracking error vector, $r_{x}(t), r_{y}(t)$ are filtered tracking error variables defined as

$$
r_{x}=\dot{\tilde{x}}+\mu \tilde{x} \quad r_{y}=\dot{\tilde{y}}+\mu \tilde{y}
$$

$\mu$ is a positive constant scalar control gain, and $\dot{\tilde{x}}(t), \dot{\tilde{y}}(t)$ represent the time derivative of $\tilde{x}(t), \tilde{y}(t)$ where $\tilde{x}(t), \tilde{y}(t), \tilde{\theta}(t)$ denote the dif-

${ }^{2}$ While the actual configuration space for the problem is $\Re^{2} \times S^{1}$, for purposes of control design and stability analysis, the configuration space can be treated as $\Re^{3}$. 
ference between the actual position/orientation and the reference position/orientation of the SV as follows:

$$
\tilde{x}=x_{c}-x_{r c} \quad \tilde{y}=y_{c}-y_{r c} \quad \tilde{\theta}=\theta-\theta_{r}
$$

and $x_{r c}(t), y_{r c}(t), \theta_{r}(t)$ are generated by the reference generator defined in (12). By taking the time derivative of (13), and using (1)-(3), (10)-(15), we can rewrite the open-loop tracking error dynamics in the following advantageous form:

$$
\begin{aligned}
\dot{w} & =u^{T} J^{T} z+f \\
\dot{z} & =u \\
\dot{u}_{1} & =-\dot{v}_{3 r}+\tau_{1}
\end{aligned}
$$

where the auxiliary $2 \times 1$ control signal $u(t)=\left[\begin{array}{ll}u_{1}(t) & u_{2}(t)\end{array}\right]^{T}$ is related to the open-loop tracking error variables defined in (16) according to the following globally invertible transformation:

$$
u=T^{-1}\left[\begin{array}{l}
F_{1} \\
v_{3}
\end{array}\right]-\Pi \quad\left[\begin{array}{l}
F_{1} \\
v_{3}
\end{array}\right]=T(u+\Pi)
$$

the $2 \times 2$ matrix $T(\cdot)$ and the auxiliary $2 \times 1$ vector $\Pi(\cdot)$ are defined as follows:

$$
\begin{aligned}
T & =\left[\begin{array}{cc}
r_{x} \sin \theta-r_{y} \cos \theta & 1 \\
1 & 0
\end{array}\right] \\
\Pi= & {\left[\begin{array}{c}
v_{3 r} \\
F_{1 r} \cos z_{1}+\frac{1}{m} Y_{v 2}\left(\dot{y}_{r c} \cos \theta_{r}-\dot{x}_{r c} \sin \theta_{r}\right) \\
\cdot \sin z_{1}-\mu\left(v_{1}-\dot{x}_{r c} \cos \theta-\dot{y}_{r c} \sin \theta\right) \\
+\frac{Y_{v 3}}{m} v_{3 r} \sin z_{1}
\end{array}\right] }
\end{aligned}
$$

$J$ is a $2 \times 2$ skew-symmetric matrix defined as follows:

$$
J=\left[\begin{array}{rr}
0 & -1 \\
1 & 0
\end{array}\right]
$$

and the auxiliary scalar signal $f(\cdot)$ is defined as follows:

$$
\begin{aligned}
f= & 2\left(v_{3 r} z_{2}-F_{1 r} \sin z_{1}+\mu(\sin \theta \dot{\tilde{x}}-\cos \theta \dot{\tilde{y}})\right) \\
& +\frac{2}{m} Y_{v 2}\left(\left(\dot{y}_{r c} \cos \theta_{r}-\dot{x}_{r c} \sin \theta_{r}\right) \cos z_{1}-v_{2}\right) \\
& +\frac{2 Y_{v 3} v_{3 r}}{m}\left(\cos z_{1}-1\right) .
\end{aligned}
$$

Remark 1: The open-loop tracking error system given in (16) is in terms of the subsequently designed control inputs $u_{2}(t)$ and $\tau_{1}(t)$. From $u_{2}(t)$ and $\tau_{1}(t)$, we can utilize (8), (9), and (17) to recover the original control inputs $F(t)$ and $\tau(t)$.

Remark 2: Based on the definition of $r_{x}(t)$ and $r_{y}(t)$ given in (14), standard arguments [5] can be utilized to prove that: i) if $r_{x}(t), r_{y}(t) \in$ $\mathcal{L}_{\infty}$ then $x_{c}(t), \tilde{x}(t), y_{c}(t), \tilde{y}(t) \in \mathcal{L}_{\infty}$, and ii) if $r_{x}(t), r_{y}(t)$ are GUUB, then $x_{c}(t), \tilde{x}(t), y_{c}(t), \tilde{y}(t)$ are GUUB.

\section{CONTROL DEVELOPMENT}

Our control objective is to design a controller that exponentially forces the tracking error to a neighborhood about zero that can be made arbitrarily small (i.e., GUUB). To this end, we define a $2 \times 1$ auxiliary error vector $\tilde{z}(t)$ as the difference between the subsequently designed $2 \times 1$ auxiliary signal $z_{d}(t)$ and the transformed variable $z(t)$ defined in (13) as follows:

$$
\tilde{z}=\left[\begin{array}{ll}
\tilde{z}_{1} & \tilde{z}_{2}
\end{array}\right]^{T}=z_{d}-z .
$$

In addition, we define an auxiliary error signal $\eta(t)$ as the difference between the subsequently designed auxiliary signal $u_{d 1}(t)$ and the auxiliary signal $u_{1}(t)$ defined in (17) as shown below

$$
\eta=u_{d 1}-u_{1} \text {. }
$$

\section{A. Control Formulation}

Based on the structure of the open-loop error system given by (16) and the subsequent stability analysis, we design the auxiliary signals $u_{d 1}(t)$ and $u_{2}(t)$ as follows:

$$
\left[\begin{array}{ll}
u_{d 1} & u_{2}
\end{array}\right]^{T}=u_{a}-k_{2} z
$$

where the $2 \times 1$ auxiliary control vector $u_{a}(t)$ is defined as

$$
u_{a}=\left(\frac{k_{1} w+f}{\delta_{d}^{2}}\right) J z_{d}+\Omega_{1} z_{d}
$$

the $2 \times 1$ auxiliary vector $z_{d}(t)$ is defined by the following oscillator-like relationship:

$$
\dot{z}_{d}=\frac{\dot{\delta}_{d}}{\delta_{d}} z_{d}+\left(\frac{k_{1} w+f}{\delta_{d}^{2}}+w \Omega_{1}\right) J z_{d} \quad z_{d}^{T}(0) z_{d}(0)=\delta_{d}^{2}(0)
$$

the auxiliary terms $\Omega_{1}(t), \delta_{d}(t)$ are scalars which are defined as follows:

$$
\begin{aligned}
\Omega_{1} & =k_{2}+\frac{\dot{\delta}_{d}}{\delta_{d}}+\frac{k_{1} w^{2}+w f}{\delta_{d}^{2}} \\
\delta_{d} & =\gamma_{0} \exp \left(-\gamma_{1} t\right)+\varepsilon_{1}
\end{aligned}
$$

where $k_{1}, k_{2}, \gamma_{0}, \gamma_{1}, \varepsilon_{1}$ are positive, constant scalar design parameters, and $f(\cdot)$ was defined in (21). Based on (16) and the subsequent stability analysis, we design the control torque input $\tau_{1}(t)$ given in (9) as follows:

$$
\tau_{1}=\dot{u}_{d 1}+\dot{v}_{3 r}+k_{3} \eta-w z_{2}+\tilde{z}_{1}
$$

where $\dot{u}_{d 1}(t)$ denotes the time derivative of $u_{d 1}(t)$ defined in (24) [see the Appendix for an explicit expression for $\left.\dot{u}_{d 1}(t)\right]$.

Remark 3: Motivation for the structure of (26) is obtained by taking the time derivative of $z_{d}^{T} z_{d}$ as follows:

$$
\begin{aligned}
\frac{d}{d t}\left(z_{d}^{T} z_{d}\right) & =2 z_{d}^{T} \dot{z}_{d} \\
& =2 z_{d}^{T}\left(\frac{\dot{\delta}_{d}}{\delta_{d}} z_{d}+\left(\frac{k_{1} w+f}{\delta_{d}^{2}}+w \Omega_{1}\right) J z_{d}\right)
\end{aligned}
$$

where (26) has been utilized. After noting that the matrix $J$ of (20) is skew symmetric, we can rewrite (30) as follows:

$$
\frac{d}{d t}\left(z_{d}^{T} z_{d}\right)=2 \frac{\dot{\delta}_{d}}{\delta_{d}} z_{d}^{T} z_{d}
$$

As result of the selection of the initial conditions given in (26), it is easy to verify that

$$
z_{d}^{T} z_{d}=\left\|z_{d}\right\|^{2}=\delta_{d}^{2}
$$

solves the differential equation given in (31). The relationship given by (32) will be used during the subsequent error system development and stability analysis.

\section{Closed-Loop ERror System DeVelopment}

To facilitate the closed-loop error system development for $w(t)$, we inject the auxiliary control input $u_{d 1}(t)$ by adding and subtracting the 
term $u_{d 1} z_{2}$ to the right side of the open-loop dynamic expression for $w(t)$ given in (16) and then utilizing (23) to obtain the following expression:

$$
\dot{w}=\left[\begin{array}{ll}
u_{d 1} & u_{2}
\end{array}\right] J^{T} z-\eta z_{2}+f .
$$

After substituting (24) for $\left[\begin{array}{ll}u_{d 1} & u_{2}\end{array}\right]$, adding and subtracting $u_{a}^{T} J_{z_{d}}$ to the resulting expression, utilizing (22), and exploiting the skew symmetry of $J$ defined in (20), we can rewrite the dynamics for $w(t)$ as follows:

$$
\dot{w}=-u_{a}^{T} J z_{d}+u_{a}^{T} J \tilde{z}-\eta z_{2}+f
$$

where we have utilized the fact that $J^{T}=-J$. Finally, by substituting (25) for only the first occurrence of $u_{a}(t)$ in (34) and then utilizing the equality given by (32), the skew symmetry of $J$ defined in (20), and the fact that $J^{T} J=I_{2}$ (Note that $I_{2}$ denotes the standard $2 \times 2$ identity matrix), we obtain the final expression for the closed-loop error system for $w(t)$ as follows:

$$
\dot{w}=-k_{1} w+u_{a}^{T} J \tilde{z}-\eta z_{2} .
$$

To determine the closed-loop error system for $\tilde{z}(t)$, we take the time derivative of (22), substitute (26) for $\dot{z}_{d}(t)$, and then substitute (16) for $\dot{z}(t)$ to obtain

$$
\begin{aligned}
\dot{\tilde{z}}=\frac{\dot{\delta}_{d}}{\delta_{d}} z_{d}+\left(\frac{k_{1} w+f}{\delta_{d}^{2}}+w \Omega_{1}\right) & J_{z_{d}} \\
& -\left[\begin{array}{ll}
u_{d 1} & u_{2}
\end{array}\right]^{T}+\left[\begin{array}{ll}
\eta & 0
\end{array}\right]^{T}
\end{aligned}
$$

where the auxiliary control input $u_{d 1}(t)$ was injected by adding and subtracting $\left[\begin{array}{ll}u_{d 1} & 0\end{array}\right]^{T}$ to the right side of (36) and then (23) was utilized. After substituting (24) for $\left[\begin{array}{ll}u_{d 1} & u_{2}\end{array}\right]^{T}$, and then substituting (25) for $u_{a}(t)$ in the resulting expression, we can rewrite the expression given by (36) as follows:

$$
\dot{\tilde{z}}=\frac{\dot{\delta}_{d}}{\delta_{d}} z_{d}+w \Omega_{1} J z_{d}-\Omega_{1} z_{d}+k_{2} z+\left[\begin{array}{ll}
\eta & 0
\end{array}\right]^{T} .
$$

After substituting (27) for only the second occurrence of $\Omega_{1}(t)$ in (37) and using the fact that $J J=-I_{2}$, we can cancel common terms and then rearrange the resulting expression to obtain

$$
\dot{\tilde{z}}=-k_{2} \tilde{z}+w J\left[\left(\frac{k_{1} w+f}{\delta_{d}^{2}}\right) J z_{d}+\Omega_{1} z_{d}\right]+\left[\begin{array}{ll}
\eta & 0
\end{array}\right]^{T}
$$

where (22) was utilized. Finally, since the bracketed term in (38) is equal to $u_{a}(t)$ defined in (25), we can obtain the final expression for the closed-loop error system for $\tilde{z}(t)$ as follows:

$$
\dot{\tilde{z}}=-k_{2} \tilde{z}+w J u_{a}+\left[\begin{array}{ll}
\eta & 0
\end{array}\right]^{T} .
$$

To develop the closed-loop error system for $\eta(t)$, we take the time derivative of (23), substitute (16) for $\dot{u}_{1}(t)$, and then rearrange the resulting expression to obtain

$$
\dot{\eta}=\dot{u}_{d 1}+\dot{v}_{3 r}-\tau_{1}
$$

After substituting for the auxiliary control torque input $\tau_{1}(t)$ given in (29), we obtain the closed-loop error system for $\eta(t)$ as follows:

$$
\dot{\eta}=-k_{3} \eta+w z_{2}-\tilde{z}_{1}
$$

\section{STABILITy ANALYSis}

Theorem 1: Given the closed-loop system of (35), (39), and (41), the position/orientation tracking error defined in (14) and (15) is GUUB in the sense that

$$
|\tilde{x}(t)|,|\tilde{y}(t)|,|\tilde{\theta}(t)| \leq \beta_{0} \exp \left(-\lambda_{0} t\right)+\beta_{1} \varepsilon_{1}
$$

where $\beta_{0}, \beta_{1}$, and $\lambda_{0}$ are positive constant scalars, and $\varepsilon_{1}$ was originally defined in (28).

Proof: To prove Theorem 1, we define a nonnegative, scalar function, denoted by $V(t)$, as follows:

$$
V=\frac{1}{2} w^{2}+\frac{1}{2} \eta^{2}+\frac{1}{2} \tilde{z}^{T} \tilde{z} .
$$

After taking the time derivative of (43) and making the appropriate substitutions from (35), (39), and (41), we obtain the following expression:

$$
\begin{aligned}
& \dot{V}=w\left[-k_{1} w+u_{a}^{T} J \tilde{z}-\eta z_{2}\right]
\end{aligned}
$$

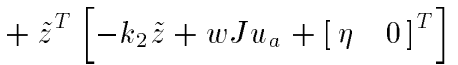

$$
\begin{aligned}
& +\eta\left[-k_{3} \eta+w z_{2}-\tilde{z}_{1}\right] \text {. }
\end{aligned}
$$

After utilizing the fact that $J^{T}=-J$, canceling common terms, and utilizing (43), we can upper bound $\dot{V}(t)$ as follows:

$$
\dot{V} \leq-2 \min \left\{k_{1}, k_{2}, k_{3}\right\} V .
$$

Standard arguments can now be employed to solve the differential inequality given in (44) as follows:

$$
V(t) \leq \exp \left(-2 \min \left\{k_{1}, k_{2}, k_{3}\right\} t\right) V(0) .
$$

Finally, based on (43), the expression given in (45) can be rewritten as

$$
\|\Psi(t)\| \leq \exp \left(-\min \left\{k_{1}, k_{2}, k_{3}\right\} t\right)\|\Psi(0)\|
$$

where the $4 \times 1$ vector $\Psi(t)$ is defined as

$$
\Psi=\left[\begin{array}{lll}
w & \eta & \tilde{z}^{T}
\end{array}\right]^{T} .
$$

From (46) and (47), it is straightforward to see that $w(t), \eta(t), \tilde{z}(t) \in$ $\mathcal{L}_{\infty}$. After utilizing (22), (32), and the fact that $\tilde{z}(t), \delta_{d}(t) \in \mathcal{L}_{\infty}$, we can conclude that $z(t), z_{d}(t) \in \mathcal{L}_{\infty}$. From the fact that $z(t), w(t) \in$ $\mathcal{L}_{\infty}$, we can use the inverse transformation of (13), given as follows:

$$
\begin{array}{r}
{\left[\begin{array}{c}
r_{x} \\
r_{y} \\
\tilde{\theta}
\end{array}\right]=\left[\begin{array}{ccc}
\frac{1}{2} \sin \theta & -\frac{Y_{v 3}}{m} \sin \theta & \frac{1}{2}(\tilde{\theta} \sin \theta+2 \cos \theta) \\
-\frac{1}{2} \cos \theta & \frac{Y_{v 3}}{m} \cos \theta & -\frac{1}{2}(\tilde{\theta} \cos \theta-2 \sin \theta) \\
0 & 1 & 0
\end{array}\right]} \\
\cdot\left[\begin{array}{c}
w \\
z_{1} \\
z_{2}
\end{array}\right]
\end{array}
$$

to conclude that $r_{x}(t), r_{y}(t), \tilde{\theta}(t) \in \mathcal{L}_{\infty}$. Based on the fact that $r_{x}(t)$, $r_{y}(t), \tilde{\theta}(t) \in \mathcal{L}_{\infty}$, and the fact that the reference trajectory is selected so that $x_{r c}(t), y_{r c}(t), \theta_{r}(t), \dot{x}_{r c}(t), \dot{y}_{r c}(t), \dot{\theta}_{r}(t) \in \mathcal{L}_{\infty}$, we can utilize (14), (15), and Remark 2 to conclude that $\dot{x}_{c}(t), \dot{y}_{c}(t), x_{c}(t), y_{c}(t)$, $\theta(t) \in \mathcal{L}_{\infty}$. From (1) and the fact that $\dot{x}_{c}(t), \dot{y}_{c}(t) \in \mathcal{L}_{\infty}$, we can conclude that $v_{1}(t), v_{2}(t) \in \mathcal{L}_{\infty}$. Using the fact that $z(t), \dot{x}_{c}(t), \dot{y}_{c}(t)$, $v_{1}(t), v_{2}(t) \in \mathcal{L}_{\infty}$, we can conclude that $f(\cdot), T(\cdot), \Pi(\cdot) \in \mathcal{L}_{\infty}$ from (21), (18) and (19). Based on these facts, we can now utilize (23)-(28), to show that $u_{d 1}(t), u_{a}(t), \dot{z}_{d}(t), \Omega_{1}(t), u_{1}(t), u_{2}(t) \in \mathcal{L}_{\infty}$. From (11), (17), and (18), we can now conclude that $F_{1}(t), \dot{\theta}(t), v_{3}(t) \in$ $\mathcal{L}_{\infty}$. Based on the previous facts, it is easy to show that $\dot{u}_{d 1}(t) \in \mathcal{L}_{\infty}$ (see the Appendix), and hence, from (29) we can conclude that $\tau_{1}(t) \in$ $\mathcal{L}_{\infty}$. Since $v_{1}(t), v_{3}(t), F_{1}(t), \tau_{1}(t) \in \mathcal{L}_{\infty}$, we can conclude from (8) and (9) that $\tau(t), F(t) \in \mathcal{L}_{\infty}$. We can now employ standard signal chasing arguments to conclude that all of the remaining signals in the control and the system remain bounded during closed-loop operation. 
To prove (42), we first show that $z(t)$ defined in (13) is GUUB by applying the triangle inequality to (22), and hence, obtain the following bound for $z(t)$

$$
\begin{aligned}
\|z\| \leq & \|\tilde{z}\|+\left\|z_{d}\right\| \\
\leq & \exp \left(-\min \left\{k_{1}, k_{2}, k_{3}\right\} t\right)\|\Psi(0)\| \\
& +\gamma_{0} \exp \left(-\gamma_{1} t\right)+\varepsilon_{1}
\end{aligned}
$$

where (28), (32), (46), and (47) have been utilized. The main result given by (42) can now be directly obtained from Remark 2, (14), (46)-(49).

\section{SETPOINT EXTENSION}

Since the only restriction placed on the desired trajectory is that the reference generator remain bounded, the position/orientation tracking problem reduces to the position and orientation regulation problem. That is, if the control objective is targeted at the regulation problem, the desired position and orientation vector, denoted by the triplet $q_{r}=$ $\left(x_{r c}, y_{r c}, \theta_{r}\right)$, becomes an arbitrary desired constant vector. Based on the fact that $q_{r}$ is now defined as a constant vector, it is straightforward to see that $F_{1 r}(t)$, and $v_{3 r}(t)$ previously defined in (12) equal zero. Moreover, $f(\cdot)$ defined in (21) reduces to the following expression:

$$
f=-2\left(\frac{1}{m} Y_{v 2} v_{2}+\mu v_{2}\right),
$$

and $\Pi(\cdot)$ defined in $(19)$ reduces to

$$
\Pi=\left[\begin{array}{c}
0 \\
-\mu v_{1}
\end{array}\right] .
$$

Based on the above simplifications, it is straightforward to illustrate that the GUUB result given in Theorem 1 is also valid for the regulation problem.

\section{CONCLUSION}

In this paper, we have designed a continuous, time-varying tracking controller for an underactuated surface vessel. Through a Lyapunovbased stability analysis, we have demonstrated that: i) the position/orientation tracking error is globally exponentially forced to a neighborhood about zero that can be made arbitrarily small, and ii) a unified framework is developed that solves the regulation problem as a simplified case of the tracking control problem. In [1], we also illustrated that the proposed controller can be applied to other nonlinear underactuated systems subject to nonintegrable dynamics such as the twin rotor helicopter. Using similar techniques as illustrated with the twin rotor helicopter extension, additional systems with similar dynamics may be solved with the proposed controller. For example, in [17], Reyhanoglu et al. described a planar prismatic-prismatic-revolute (PPR) robot with an elastic joint that has similar dynamics as the examples presented in this paper. It is straightforward to illustrate that the proposed controller yields a GUUB tracking/regulation result for the PPR elastic-joint robot utilizing similar arguments presented in the twin rotor helicopter controller of [1].

\section{APPENDIX \\ CALCULATION OF $\dot{u}_{d 1}(t)$}

To calculate $\dot{u}_{d 1}(t)$, we take the time derivative of (24) and then substitute for the time derivative of $u_{a}(t)$ defined in (25) to obtain the following expression:

$$
\begin{aligned}
\dot{u}_{d 1}=-\left(\frac{k_{1} \dot{w}+\dot{f}}{\delta_{d}^{2}}\right) z_{d 2} & +2\left(\frac{\left(k_{1} w+f\right) \dot{\delta}_{d}}{\delta_{d}^{3}}\right) z_{d 2} \\
& +\dot{\Omega}_{1} z_{d 1}+\Omega_{1} \dot{z}_{d 1}-\left(\frac{k_{1} w+f}{\delta_{d}^{2}}\right) \dot{z}_{d 2}-k_{2} \dot{z}_{1}
\end{aligned}
$$

where the time derivatives of $\Omega_{1}(t)$ and $f(t)$ are explicitly given by the following expressions:

$$
\begin{aligned}
\dot{\Omega}_{1}= & \frac{\ddot{\delta}_{d}}{\delta_{d}}-\frac{\dot{\delta}_{d}^{2}}{\delta_{d}^{2}}+\frac{\left(2 k_{1} w+f\right) \dot{w}+w \dot{f}}{\delta_{d}^{2}} \\
& -2 \frac{\left(k_{1} w^{2}+w f\right) \dot{\delta}_{d}}{\delta_{d}^{3}}
\end{aligned}
$$

and

$$
\begin{aligned}
\dot{f}= & 2\left(v_{3 r} u_{2}+\dot{v}_{3 r} z_{2}-\dot{F}_{1 r} \sin z_{1}-F_{1 r} u_{1} \cos z_{1}\right) \\
& +2 \mu\left((\dot{\tilde{x}} \cos \theta+\dot{\tilde{y}} \sin \theta) v_{3}\right) \\
& -2 \mu\left(\frac{Y_{v 2}}{m}\left(\dot{y}_{c} \cos \theta-\dot{x}_{c} \sin \theta\right)\right. \\
& \left.+\frac{Y_{v 3} v_{3}}{m}+\ddot{x}_{r c} \sin \theta-\ddot{y}_{r c} \cos \theta\right) \\
+ & \frac{2 Y_{v 2}}{m}\left(y_{r c} \cos \theta_{r}-\ddot{x}_{r c} \sin \theta_{r}\right. \\
& \left.\quad-\dot{\theta}_{r}\left(\dot{y}_{r c} \sin \theta_{r}+\dot{x}_{r c} \cos \theta_{r}\right)\right) \cos z_{1} \\
& -\frac{2 Y_{v 2}}{m}\left(\left(\dot{y}_{r c} \cos \theta_{r}-\dot{x}_{r c} \sin \theta_{r}\right) u_{1} \sin z_{1}\right) \\
& -\frac{2 Y_{v 2}}{m}\left(\left(\frac{1}{m}\left(Y_{v 2} v_{2}+Y_{v 3} v_{3}\right)-v_{1} v_{3}\right)\right) \\
& +2\left(\frac{Y_{v 3} \dot{v}_{3 r}}{m}\left(\cos z_{1}-1\right)-u_{1} \frac{Y_{v 3} v_{3 r}}{m} \sin z_{1}\right)
\end{aligned}
$$

where (10)-(12), (16), and the second time derivative of (15) was utilized. Based on the definition of $\delta_{d}(t)$ given in (28), the fact that $z(t)$, $\dot{z}(t), \dot{\tilde{\theta}}(t), u(t), \dot{\tilde{x}}(t), \dot{\tilde{y}}(t), \dot{f}(t), \dot{\Omega}_{1}(t), \dot{w}(t), \dot{z}_{d}(t), w(t), f(t), z_{d}(t)$, $u_{d 1}(t), \eta(t) \in \mathcal{L}_{\infty}$ (see Theorem 1 ), and the fact that the reference trajectory is selected so that $x_{r c}(t), y_{r c}(t), \theta_{r}(t), \dot{x}_{r c}(t), \dot{y}_{r c}(t), \dot{\theta}_{r}(t)$, $\ddot{x}_{r c}(t), \ddot{y}_{r c}(t), \ddot{\theta}_{r}(t), \dot{v}_{3 r}(t) \in \mathcal{L}_{\infty}$, it is straightforward to see from (50)-(52) that $\dot{u}_{d 1}(t) \in \mathcal{L}_{\infty}$.

\section{ACKNOWLEDGMENT}

The authors would like to thank the reviewers for their constructive suggestions and a careful review of the manuscript.

\section{REFERENCES}

[1] A. Behal, D. M. Dawson, W. E. Dixon, and Y. Fang, "Tracking and regulation control of an underactuated surface vessel with nonintegrable dynamics," in Proc. 39th IEEE Conf. Decision Control, Sydney, Australia, Dec. 12-15, 2000, pp. 2150-2155.

[2] A. Bloch and S. Drakunov, "Stabilization and tracking in the nonholonomic integrator via sliding modes," Syst. Control Lett., vol. 29, no. 2 , pp. 91-100, Oct. 1996.

[3] R. Brockett, "Asymptotic stability and feedback stabilization," in Differential Geometric Control Theory, R. Brockett, R. Millman, and H. Sussmann, Eds. Boston, MA: Birkhauser, 1983.

[4] C. Canudas de Wit and O. Sordalen, "Exponential stabilization of mobile robots with nonholonomic constraints," IEEE Trans. Automat. Contr. vol. 37, pp. 1791-1797, Nov. 1992.

[5] D. M. Dawson, J. Hu, and T. Burg, Nonlinear Control of Electric Machinery. New York: Marcel Dekker, 1998.

[6] W. E. Dixon, D. M. Dawson, E. Zergeroglu, and F. Zhang, "Robust tracking and regulation control for mobile robots," Int. J. Robust Nonlinear Control: Special Issue Control Underact. Nonlinear Syst., vol. 10, pp. 199-216, 2000

[7] W. E. Dixon, D. M. Dawson, F. Zhang, and E. Zergeroglu, "Global exponential setpoint control of mobile robots," in Proc. IEEE/ASME Int. Conf. Advanced Intelligent Mechatronics, Atlanta, GA, Sept. 1999, pp. 683-688.

[8] T. I. Fossen, Guidance and Control of Ocean Vehicles. Chichester, U.K.: Wiley, 1994. 
[9] J. M. Godhavn, "Nonlinear tracking of underactuated surface vessels," in Proc. 35th Conf. Decision Control, Kobe, Japan, Dec. 1996.

[10] Z. Jiang and H. Nijmeijer, "Tracking control of mobile robots: A case study in backstepping," Automatica, vol. 33, no. 7, pp. 1393-1399, 1997.

[11] _ - "A recursive technique for tracking control of nonholonomic systems in the chained form," IEEE Trans. Automat. Contr., vol. 44, pp. 265-279, Feb. 1999.

[12] R. McCloskey and R. Murray, "Exponential stabilization of driftless nonlinear control systems using homogeneous feedback," IEEE Trans. Automat. Contr., vol. 42, pp. 614-628, May 1997.

[13] R. Pettersen and O. Egeland, "Exponential stabilization of an underactuated surface vessel," Modeling, Ident. Control, vol. 18, no. 3, p. 239, July 1997.

[14] K. Y. Pettersen and O. Egeland, "Robust control of an underactuated surface vessel with thruster dynamics," in Proc. Amer. Control Conf., Albuquerque, NM, June 1997, pp. 3411-3416.

[15] K. Y. Pettersen and H. Nijmeijer, "Global practical stabilization and tracking for an underactuated ship-A combined averaging and backstepping approach," in Proc. IFAC Conf. System Structure Control, Nantes, France, July 1998, pp. 59-64.

[16] - "Tracking control of an underactuated surface vessel," in Proc. 37th IEEE Conf. Decision Control, Tampa, FL, Dec. 1998, pp. 4561-4566.

[17] M. Reyhanoglu, A. Schaft, N. H. McClamroch, and I. Kolmanovsky, "Dynamics and control of a class of underactuated mechanical systems," IEEE Trans. Automat. Contr., vol. 44, pp. 1663-1670, Sept. 1999.

[18] C. Samson, "Velocity and torque feedback control of a nonholonomic cart," in Proc. Int. Workshop Adaptive Nonlinear Control: Issues Robotics, Grenoble, France, 1990.

[19] — , "Control of chained systems application to path following and time-varying point-stabilization of mobile robots," IEEE Trans. Automat. Contr., vol. 40, pp. 64-77, Jan 1997.

[20] F. Y. Zheng, Ed., Recent Trends in Mobile Robots, Singapore: World Scientific, 1993.

\section{Lyapunov-Based Switching Supervisory Control of Nonlinear Uncertain Systems}

David Angeli and Edoardo Mosca

\begin{abstract}
The problem of controlling nonlinear noisy systems affected by parametric uncertainties is approached via the introduction of a supervisor which switches on, in feedback to the plant, one controller selected from a finite set of predesigned controllers. A Lyapunov-based falsification criterion allows one to ensure robust stability in the presence of uncertain constant parameters and exogenous bounded disturbances.
\end{abstract}

\section{INTRODUCTION}

One of the well-established approaches for dealing in control with plant model uncertainty is the introduction of adaptation in the feedback loop. However, conventional continuous adaptation is not always capable of performing satisfactorily. This may be particularly true whenever the plant switches among different modes of operation or if closed-loop signals are not sufficiently exciting. In both circumstances undesirable transients may typically arise due to slow adaptation.

Manuscript received February 7, 2000; revised January 8, 2001 and December 3, 2001. Recommended by Associate Editor E. Feron. This work was supported in part by MURST via the Project "Algoritmi e architetture per l'identificazione e il controllo di sistemi industriali."

The authors are with the Dipartimento Sistemi e Informatica, University of Firenze, 50139 Firenze, Italy. (e-mail: angeli@dsi.unifi.it; mosca@ dsi.unifi.it). Publisher Item Identifier S 0018-9286(02)02830-1.
In recent years, switching supervisory control (SSC) has emerged as an alternative approach for tackling the problem with the appealing feature of resembling an adaptive version of classic gain-scheduling control [3], [9], [6], [11]-[13] which has been successful in so many applications. As a matter of fact, SSC aims at extending gain-scheduling control to cases where the supervisor has no full information on the current dynamical behavior of the plant to be controlled.

If the unknown plant belongs to a certain prespecified set of models, the idea is to have a set of candidate controllers $\mathcal{C}_{i}$, usually finite, so that each plant model performs "satisfactorily" when controlled by at least one of the $\mathcal{C}_{i}$ s. Then, a suitably designed supervisory unit takes care of orchestrating the switching among the candidate controllers so as to ensure closed loop stability in the face of uncertainty or even in the presence of a sufficiently slow drifting of the uncertain parameters. A related area of research deals with robust (rather than adaptive) stabilization of uncertain plants by means of switching controllers [2].

Within SSC the fundamental problems to be tackled are the following two: controller falsification, and inference of candidate loop behavior. Controller falsification [13] deals with the problem of finding criteria according to which the supervisor decides whether the acting controller is adequate or not. Inference of candidate loop behavior [10] deals with the problem of inferring the behavior of the potential loop made up by a candidate controller connected in feedback to the uncertain plant on the grounds of data taken from the currently operating loop. To date, most of the algorithms in the literature on SSC rely on the evaluation of output prediction errors which, properly filtered and treated, are used as "performance signals" for both controller falsification and inference of candidate loop behavior, [5], [8], [10]. The main contribution of this note is to keep those two issues well separated and present Lyapunov-based falsification criteria applicable both to linear and nonlinear plants.

\section{PROBLEM Formulation}

Consider a discrete-time uncertain nonlinear system of the following general form:

$$
x(t+1)=f(x(t), u(t), \theta)
$$

with states $x \in \mathbb{R}^{n}$, inputs $u \in \mathcal{U} \subset \mathbb{R}^{p}$ and unknown parameters $\theta$ taking value in some totally limited space $\Theta$. Our aim is to design a controller in the form of a state feedback suitably managed by a supervisory unit, in such a way that the resulting closed-loop system be globally asymptotically stable, irrespective of plant uncertainties. Supervisory logic and simulation results consider the case of possibly time-varying parameters, though, for the sake of simplicity the analysis will be carried out assuming $\theta$ constant. The supervisory unit is devised so as to deal with possibly large uncertainties by adaptively selecting a suitable feedback gain among a finite family of predesigned controllers. The crucial assumption in this respect will be the existence of a finite cover for $\Theta$

$$
\Theta \subset \bigcup_{i=1 \cdots N} \Theta_{i}
$$

and of a family of Lyapunov functions $\left\{V_{i}(x)\right\}_{i=1} \cdots N$ and controllers $\left\{k_{i}(x): \mathbb{R}^{n} \rightarrow \mathcal{U}\right\}_{i=1: N}$ with the property that

$$
V_{i}\left(f\left(x, k_{i}(x), \theta\right)\right)-V_{i}(x) \leq-\rho(|x|), \quad \forall \theta \in \Theta_{i}, \forall x
$$

for some positive-definite function $\rho: \mathbb{R}_{\geq 0} \rightarrow \mathbb{R}_{\geq 0}$. As usual, in the context of nonlinear control, we assume $V_{i}(x)$ to be positive definite and radially unbounded, (not necessarily continuous except at the 